\title{
Knowledge, Attitude and Practice Towards Modern Family Planning Methods Among Mothers of Reproductive Age Group in Wonago Health Center, Southern Ethiopia
}

\author{
Kaleab Tesfaye Tegegne (BSCPH, MPH) \\ Department of Public Health, Hawassa College Of Health Science, Hawassa, Ethiopia
}

\begin{abstract}
Back ground: The continuing growth of the world population has become an urgent global problem. Ethiopia like most countries in Sub-Saharan Africa is experiencing rapid population growth. Currently, the country's is population is growing at a rate of $3 \%$ is one of the largest rates in the world and if it continues unabated, the population will have doubled in 23 years , preventing any gain in the national development effort.

Objectives: The objective of this study was to determine the knowledge, attitude and practice of mothers towards family planning in Wonago health center.

Methods: cross- sectional study was conducted from April 15, 2004 E.C to June 10, 2004 E.C., in Wonago health center, Gedeo Zone, SNNPR, Ethiopia. A total of 251 study subjects were selected by systematic sampling technique from mothers of child bearing age (15-49) who attended Wonago health center for any services. Data was collected by using interviewees. Chi-square test and odds ratio were used to assess the presence and strength of association between independent and dependent variables.

Result: Out of total 251 women of reproductive age group who participated in the study 206(82.3\%) had ever heard about FP, of which 202(98\%) knew at least one method of FP and 116(59\%) were using some method of FP at time of the study .the information about FP among reproductive age women of urban dwellers is thirteen times higher than rural dwellers $\mathrm{OR}=13.74(4.747,39.79) \mathrm{p}$-value 0.001 ,educational status showed statistically significant difference p-value 0.001 and women who possessed TV two times more likely had ever practiced FP methods than those who did not OR=2.599(1.441,4.6899), P-value 0.001.

Conclusion and recommendation: In the study the major source of information on FP is from the health institution, majority of the respondents knew at least one type of modern FP methods and socio demographic factors like residence educational status are associated with information about FP and ever practice of it .community should be encouraged to use long term contraceptives so as to space their family and decrease the frequent visit to health institution
\end{abstract}

Keywords: knowledge, attitude, practice, modern family planning

DOI: $10.7176 / \mathrm{JNSR} / 9-19-02$

Publication date:October $31^{\text {st }} 2019$

\section{Introduction}

Family planning is a decision made by an individual or couple about how many children one would like to have when to start having children, when to stop having children and how long to rest between each pregnancy. The decision has to be made freely without any coercion, after the individual has been fully informed about the benefits of planning of family size, the methods one can use, the relative advantages of each of the methods as well as the expected side effects of all the methods described (2)

Ethiopia is considered to be one of most populous countries in Africa following Nigeria and Egypt. The growth rate, estimated at less than $0.5 \%$ in 1900 , increased to $2 \%$ in the $1950 \mathrm{~s}$, to $2.5 \%$ during the late $1970 \mathrm{~s}$, and to $2.9 \%$ in 1984. According to the first census, in 1900 the population of Ethiopia was estimated to be 11.0million and by 1994 it was about 53.5 million. Between 1999 and mid 2000, the population of Ethiopia was estimated to be 62 million with annual growth rate of $3 \%$ (3)

The continuing growth of the world population has become an urgent global problem. Ethiopia like most countries in Sub-Saharan Africa, is experiencing rapid population rate of 3\% is one of the highest rates in the world and if it continuous unabated, the population will have doubled in 23 years, preventing any gain in the national development effort (7)

Access to family planning is an important challenge in Ethiopia. With better primary health care, childhood immunization, infant mortality is falling, so families increasingly want to limit the numbers of children they have. The shift to the smaller family size is highly the important driver of development, known as the, demographic transition. When a family two or more children, all of whom are likely to survive, they are able to invest in the children's nutrition, health and education, in a way that is impossible for most, families with nine or ten children. The desire to have smaller families is driven by a combination of rising income, improved life expectancy, lower infant mortality, better education and increased savings, as well as changing cultured and social norms. Today Ethiopia and Germany have roughly the same number of people (around 82 million. But unless something changes, 
by 2050 Ethiopia is projected to more than double its population to 174 million, while over the same period Germany's population is likely to decline to 72 million. The cause is simple: Ethiopia's total fertility rate of 5.4 is four times greater than that of Germany's rate of 1.4 (20).

In 1971, the Ethiopian population was estimated at 25 million and the growth rate was at 2.5 percent. Although population problems are suspected to have contributed to the health based economic problems of the country in the recent years, no large scale have been conducted in this area and the government has not formulated an official police on family planning. During 1970-71, the first survey of knowledge, attitude and practice of family planning in Ethiopia was carried out under the sponsorship of the institute of Ethiopian studies of Haile sellase first University. This study was designed to provide a rudimentary data base for the national development and health planning (8) The study help to Identify the factors affecting KAP towards FP so as to inform health service managers to take measures. and Inform the better ways through which KAP towards FP will be improved. and this study serves as reference for those who will conduct same study in the area.

\section{Subjects and Methods}

\subsection{Study area and period}

This study was conducted in wonago Health center from April 15, 2004 E.C to June10, 2004 E.C. Wonago is a Capital of Wonago Woreda which is one of the six woredas in Gedeo Zone, SNNPR, Ethiopia. It shares boundaries with Tumata Chirata kebele in the north, Sugale kebele in the south and east, and Bale bukisa kebele in the west. The town lies in international asphalt road line that connects Addis Ababa with Nairobi, Kenya. It is found 375 $\mathrm{km}$ from Addis Ababa, $98 \mathrm{~km}$ from Hawassa, capital city of SNNPR and 12km from Dilla, capital of Gedeo Zone. It covers a total area of 68 hectares having a total population of 16,451as the 2003 E.C report of Wonago municipality.

The study was conducted from April 15, 2004 E.C to June 10, 2004 E.C

\subsection{Study design}

Institution based cross-sectional study design was conducted

\subsection{Study population}

Selected women in reproductive age group (15-49) who attended wonago Health center for any services during data collection period.

\subsection{Inclusion and Exclusion criteria}

\subsection{Inclusion Criteria}

Selected woman of reproductive age (15-49) who was not seriously ill, had no hearing and speaking problem and mentally healthy.

\subsection{Exclusion criteria}

Selected woman who was seriously ill, had hearing and speaking problem and mental illness

\subsection{Sample size and Sampling technique}

Single population proportion formula was used to calculate sample size with confidence interval of $95 \%$ assuming $5 \%$ marginal error and $\alpha=5 \%$. Proportion of sample size determination was used from Ethiopia Demographic and health survey conducted in 2011 on KAP of the reproductive age women on family planning methods showed that about $97.2 \%$ women have heard of any family planning method, $19.6 \%$ used any of the method.

$$
\begin{aligned}
& \mathrm{n}=\left(\underline{\mathrm{Z}}_{2} / 2\right)^{2}(\mathrm{p})(1-\mathrm{p}) \\
& \mathrm{w}^{2} \\
& \text { Where } \\
& \mathrm{n}=\text { sample size } \\
& \mathrm{z}=\text { confidence coefficient } \\
& \mathrm{w}=\text { marginal error } \\
& \mathrm{p}=\text { proportions }
\end{aligned}
$$

From the DHS, we had

Knowledge $\left(\mathrm{p}_{\mathrm{I}}\right)=97.2 \%$

Practice $\left(\mathrm{p}_{2}\right)=19.6 \%$

So $\mathrm{n} 1=\underline{(\mathrm{z} \propto / 2)\left(\mathrm{p}_{\mathrm{I}}\right)\left(1-\mathrm{p}_{\mathrm{I}}\right)}$ 


$$
=\frac{(1.96)^{2}(0.972)(1-0.972)}{(0.09)^{2}}=41.82 \approx 42,
$$

Considering $10 \%$ non- respondent rate it will be, $42+42 \times 15 \%=\underline{46}$

Since the source population was more than 10,000 which was 22,595 we didn't use correction formula

$$
\begin{aligned}
& \mathrm{n}_{2}=\frac{(\mathrm{zx} / 2)^{2}(\mathrm{p} 2)(1-\mathrm{p} 2)}{\mathrm{w} 2} \\
& =\frac{(1.96)^{2}(0.196)(1-0.196)}{(0.05)^{2}}=242.14 \approx
\end{aligned}
$$

242 Where $\mathrm{p}_{2}=0.196$

Considering $10 \%$ non-respondent rate $\mathrm{n}_{2}$ will bee

$242+242 \times 10 \%=242+24.2$

$=242+24.2 \approx 266.2 \approx 266$

For the sake of generalizations we used the proportion with the largest sample size i.e. practice $(\mathrm{p} 2)=19.6 \%$ $\mathrm{nf}=266$

Systematic sampling technique was used to select women of child bearing age who attended Wonago health center for any services.

The interval of the study population selected was calculated as follow:

$\mathrm{K}=\frac{N}{n} \quad$ Where:

$=\frac{1500}{266} \quad \mathrm{~K}=$ is number of interval between study subjects.

$=5.6390 \approx 6 \quad \mathrm{n}=$ sample size $(266)$

$\mathrm{N}=$ Reproductive age group women who attended health center

To get the study subjects, source population was listed 1,2,3, 1500. The first study subject was selected from first six source subjects by a lottery method which we found was second source subject. For the rest of the study subjects, every $6^{\text {th }}$ source subject will be selected i.e. $2^{\text {nd }}, 8^{\text {th }}, 14^{\text {th }}, 20^{\text {th }}$,

\subsection{Data collection procedures}

The data collection tools were structured questioner which consists knowledge, attitude and practice questions on family planning. Data was collected regarding the dependent and independent variables by interview administered questionnaire

\subsection{Data quality assurance}

Trained data collector were undertake data collection .Before the actual data collection, the questionnaire was pre tested in $5 \%$ of women in reproductive age group (15-49) in other health centers Based on the pretest, necessary modification were made on the questions and the data of the pretest was excluded in the actual data analysis

To keep the data as accurate as possible, the structured check list for data collection was used thoroughly throughout the process. Completeness of the data and possible errors was also be monitored carefully.

\subsection{Data processing and analysis}

After collection of data the responses were coded and entered using EPI info version 3.1 statistical programs. After data entry was finished, attentively checked for consistency of data entry. Data was cleaned accordingly and then exported to SPSS version 16.0 for further analysis. Then frequency distribution of dependent and independent variables was worked out. To establish association between dependent independent variables, $\mathrm{X}^{2}$ test and $\mathrm{p}$-values were used. Statistical significance was considered at $\mathrm{p}$-value less than 0.05 .

\subsection{Operational Definitions}

Attitude: ones look towards FP.

Current FP method user:-woman using any one of the modern FP methods currently Emergency contraceptive: - a kind of contraceptive indicated after unprotected sexual inter course to prevent unintended pregnancy.

Ever use of FP method: use of any method of FP at least once during the sexual life.

High income: - monthly income of birr more than 500 
Illiterate:-those respondents who did not go to school and could not read and write.

Knowledge: - awareness of the existence of family planning, its importance and effectiveness.

Low income: - monthly income of less than 500 birr.

Modern FP methods:-contraceptive methods which include OCPs, injectables, condoms, implants, IUCDs, diaphragm, vaginal foam, BTL.

Practice: - any previous history of family planning methods used.

Sexually active: - having a previous history of vaginally sexual intercourse.

Unintended pregnancy: - pregnancy which occurred without plan.

\subsection{Ethical considerations}

Ethical clearance was obtained from research and ethical committee (REC) of Rift Valley University, department of public health. The consent was obtained from Wonago health center and selected mothers were studied after purpose and procedure of the study were explained and verbal consent was obtained from all respondents and all data obtained from individual was kept confidentially by using codes instead of any personal identifier.

\section{Results}

Out of the calculated sample of 266 sample of reproductive age women 251 responded to the interview while 15 women refused to participate in the study. Analysis was based on the 251 questionnaires making the overall response rate of $94.3 \%$. Out of 251 respondents $129(51.4 \%)$ were rural dwellers and $122(48.6 \%)$ were urban dwellers. Gedeo $127(70.5 \%)$ and Amhara 41(16.3\%) were the majority of ethnic groups and followed by others. Protestant were the majority of the religious group which comprises $172(68.5 \%)$ followed by orthodox $64(25.5 \%)$ followers and the rest comprises for minority. Majority of the respondents were married $219(87.3 \%)$ and $30(12 \%)$ were single and $2(0.7 \%)$ were divorced. The majority of the respondents were house wives $125(49.8 \%)$ followed by government employed $45(17.9 \%)$ and students $33(13.4 \%)$. The majority of the respondents were illiterates $109(43.4 \%)$ followed by primary school $77(30.7 \%)$.

Out of 251 respondents who were participated in the study 150 of them responded about their monthly income while the rest refused or did not want to estimate. Of those 81 respondents (54\%) earned monthly income between 500-1000 ETB followed by 42 respondents (16.5\%) earned less than 500 ETB per month. Out of 251 respondents $77(30.5 \%)$ owned TV, $155(61.8 \%)$ owned radio. The majority of the respondents $166(66.1 \%)$ had no cattle; the rest of the respondents had at least one cattle.

\section{Family Planning Knowledge of the respondents}

Knowledge of modern FP was found to be universal; 206(82.5\%) of respondents had ever heard about FP regardless of educational level and socio economic status. The source of information for the majority $145(70.4 \%)$ from the health institution, followed by mass media and friend which accounts for 50(24.3\%) and 44(21.4\%) respectively, the rest 28(13.6\%) got from relative (see also figure 1). All except 4 respondents (1.94\%) who did not know any FP of total 206 respondents who had information on FP the rest $202(98.06 \%)$ or $80.5 \%$ of all the respondents knew at least one method of FP. Out of 202 respondents majority $178(86.4 \%)$ knew depo- provera, followed by $138(69 \%)$ knew pills, 82(39.8\%) knew nor plant ,and 81(39.3\%) knew IUCD, 69(33.5\%) knew condom and 97(47.1\%) knew two and more FP methods.

Questions were also asked about importance of FP; 192(94.1\%),165 (80.9\%) and $158(77.5 \%)$ respondents selected that FP uses for child spacing, prevention of unwanted pregnancy and for limiting family size respectively; 34 respondents selected two or more importance.

\section{Attitudes Towards Family Planning of the Respondents}

Attitude is the most difficult to measure as it is characterized in a very abstract way. To gauge the attitudes towards FP, it was noted in the study that for five questioners with three alternatives'; agree, disagree and neutral were interviewed and their response was recorded in questioners accordingly.

The result showed that $202(80.5 \%)$ respondents agreed on the fact that too large family size is harmful to overall well being of the family; out of 206 respondents who had information about FP 197(95.6\%) and 7 respondents(3.4\%) agreed and neutral respectively on question "FP improves ones standard of living." ;201women agreed on that discussion about FP is good; 172(83.9\%) agreed on "FP helps mother to regain strength before her next pregnancy.";84(40.8\%) disagreed and 79(38.3\%) agreed on that "Couple who practice FP methods will have conflict in their marriage.

\section{Family Planning Practice of the Respondents}

About $148(59 \%)$ of respondents had previously used some modern FP methods and $116(46.2 \%)$ of the respondents were using FP methods at the time of the study. Among 116 current users 93(79.5\%) were Depo-Provera users, 11(9.4\%) were Norplant users, $8(6.8 \%)$ were using IUCD, 4(4.3\%) were using pills see also figure 2 Sixteen 
(13.8\%) of ever used FP methods changed to some form of FP methods at the time of the study for the following reasons; $13(81.2 \%)$ changed for the reason of side effects, $2(12.5 \%)$ and $1(6.5 \%)$ users changed for the reason to use for the long duration and inaccessibility of the desired FP method respectively.

Among 103(37.3\%) of study subjects who had not ever used any FP method, they had different reasons for non use; lack of knowledge $48.5 \%$; and not practice of sexual intercourse and desire to have more children were share $24.3 \%$ both; and the rest $2.9 \%$ accounts for other reasons.

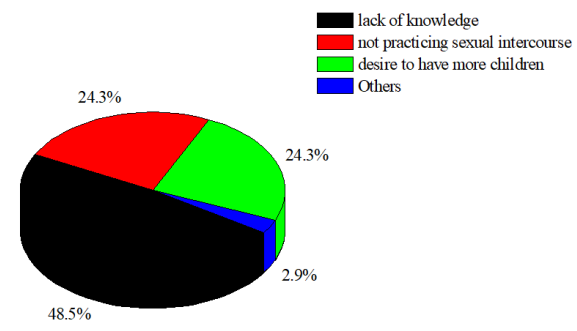

Figure 1 Reason for non use of Family planning among women of reproductive age group in Wonago health center, SNNPR, June 2012

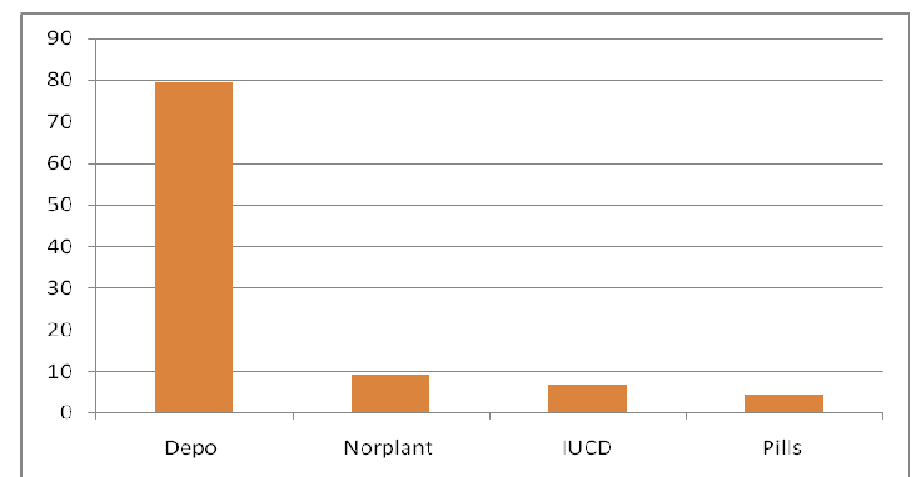

NB: percentages add up more than $100 \%$ because more than one answer was possible.

Figure 2. Percentages of different types of FP methods used at time of study among women of reproductive age group in Wonago health center, SNNPR, June 2012

\section{Factors Associated with information on Family Planning}

The information about FP among reproductive age women of urban dwellers is thirteen times higher than rural dwellers $\mathrm{OR}=13.74(4.747,39.79) \mathrm{p}$-value 0.001 . Educational status showed statistically significant difference, $p$ value 0.001 . Analysis of the family size of the study population showed that the respondents who had more than six family members were 0.3 times likely had information on FP OR=0.321(0.108,0.949) p-value 0.033.

Reproductive age women who possessed TV 25 times more likely to get information about FP as compared with those who did not possess and those who possess radio six times more to be informed on FP $\mathrm{OR}=6.242(3.026,12.87) \mathrm{p}$-value 0.001 .

Reproductive variables of women of reproductive age group by information about FP

Analysis of reproductive variable like need of additional children showed no statistically significant difference $\mathrm{p}$-value $=0.093($ see table 1$)$ 
Table 1. The association of selected socio demographic variables of the women of reproductive age by information about FP in Wonago health center, Southern Ethiopia, June 2012.

\begin{tabular}{|c|c|c|c|c|c|}
\hline \multirow[t]{2}{*}{ Variables } & \multicolumn{2}{|c|}{ Information about FP } & \multirow[t]{2}{*}{$\mathbf{X}^{2}$} & \multirow[t]{2}{*}{ OR $(95 \%$ CI) } & \multirow[t]{2}{*}{ P-value } \\
\hline & Yes & No & & & \\
\hline \multicolumn{6}{|l|}{ Residence } \\
\hline Urban & 118 & 4 & 34.62 & $13.74(4.747,39.79)$ & $0.001 *$ \\
\hline Rural & 88 & 41 & & & \\
\hline Total & 206 & 45 & & & \\
\hline \multicolumn{6}{|l|}{ Educational status } \\
\hline Illiterate & 71 & 38 & & & \\
\hline Primary school & 70 & 7 & 39.529 & & $0.001 *$ \\
\hline Secondary school & 31 & 0 & & & \\
\hline Tertiary school & 34 & 0 & & & \\
\hline Total & 206 & 45 & & & \\
\hline \multicolumn{6}{|l|}{ Family size } \\
\hline Six and less & 135 & 38 & 4.536 & $0.323(0.109,0.956)$ & $0.033 *$ \\
\hline More than six & 44 & 4 & & & \\
\hline Total & 179 & 42 & & & \\
\hline \multicolumn{6}{|l|}{ Television } \\
\hline Yes & 176 & 1 & 20.876 & $25.723(3.474,190.490)$ & $0.001 *$ \\
\hline No & 130 & 44 & & & \\
\hline Total & 206 & 45 & & & \\
\hline \multicolumn{6}{|l|}{ Radio } \\
\hline Yes & 143 & 12 & 28.578 & $6.242(3.026,12.87)$ & $0.001 *$ \\
\hline No & 63 & 33 & & & \\
\hline Total & 206 & 45 & & & \\
\hline
\end{tabular}

NB:* indicate statistically significant when OR does not cross one.

Factors Associated with ever practice of Family Planning methods

Women of reproductive age group who dwelled in urban three more likely to practice FP methods as compared to rural dwellers, $\mathrm{OR}=3.2(1.866,5.429)$ and $\mathrm{p}$-value 0.001. Educational status showed statistically significant difference and p-value 0.003 , but family size showed no statistically significant difference

Women who possessed TV two times more likely ever had practiced FP methods than those who did not $\mathrm{OR}=2.599(1.441,4.6899) \mathrm{p}$-value 0.001 and those who owned radio four times more likely had ever practiced FP methods than those who did not $\mathrm{OR}=4.023(2.346,6.898)$, p-value 0.001

Reproductive variable like number of males the women possessed and need of additional children showed no statistically significant difference on ever practice of FP methods (see table 2). 
Table 2. The association of background variables of women of reproductive age group by ever practices of FP methods in Wonago health center, Southern Ethiopia, June, 2012

\begin{tabular}{|c|c|c|c|c|c|}
\hline \multirow[t]{2}{*}{ Variable } & \multicolumn{2}{|c|}{ Ever Practiced FP Methods } & \multirow[t]{2}{*}{$\mathrm{X}^{2}$} & \multirow[t]{2}{*}{ OR (95\% CI) } & \multirow[t]{2}{*}{ P-value } \\
\hline & Yes & No & & & \\
\hline \multicolumn{6}{|l|}{ Residence } \\
\hline Urban & 89 & 33 & 19.92 & $3.2(1.866,5.429)$ & $0.001 *$ \\
\hline Rural & 59 & 70 & & & \\
\hline Total & 148 & 103 & & & \\
\hline \multicolumn{6}{|c|}{ Educational status } \\
\hline Illiterate & 51 & 58 & 14.093 & & $0.003 *$ \\
\hline Primary & 53 & 24 & & & \\
\hline Secondary & 18 & 13 & & & \\
\hline Tertiary & 26 & 8 & & & \\
\hline Total & 148 & 103 & & & \\
\hline \multicolumn{6}{|l|}{ Family size } \\
\hline Six and less & 109 & 64 & 1.626 & $0.633(0.312,1.283)$ & 0.202 \\
\hline More than six & 35 & 13 & & & \\
\hline Total & 144 & 77 & & & \\
\hline \multicolumn{6}{|l|}{ Television } \\
\hline Yes & 57 & 20 & 10.414 & $2.599(1.441,4.6899)$ & $0.001 *$ \\
\hline No & 91 & 83 & & & \\
\hline Total & 148 & 103 & & & \\
\hline \multicolumn{6}{|l|}{ Radio } \\
\hline Yes & 111 & 44 & 26.797 & $4.023(2.346,6.898)$ & $0.001 *$ \\
\hline No & 37 & 59 & & & \\
\hline Total & 148 & 103 & & & \\
\hline
\end{tabular}

NB. * indicates statistically significant when OR does not cross one.

\section{Discussion and Conclusion}

A total of 251 subjects participated in this study making response rate of $94.3 \%$. The result of our study showed that $82.4 \%$ of which urban residents are more likely to be informed on family planning than rural dwellers (96.7\%Vs 68.2\%); this result showed that urban dwellers have similar but that of the rural one is less when compared with Similar cross sectional study conducted on modern contraceptive and knowledge attitude and practice study among women of reproductive age in Bahir Dar town and per urban area showed that $98 \%$ of women in urban and $96 \%$ in per urban area had heard of modern contraceptives (14).

In this study, $80.5 \%$ of respondents can spontaneously name at least one type of family planning method. Similar cross sectional study conducted to assess the knowledge attitude and practice of the mothers towards the family planning in regions of Amhara, Oromiya, SNNPR and Tigray regions of the Ethiopia to assess the knowledge attitude and practice of women's towards the family planning showed that $84.0 \%$ of women in SNNPR reported that they had heard of family planning method(12).

In our study socio demographic variables like occupation, religion and ethnicity did not show significant association with ever practice of FP which is similar with the study conducted in Bahir Dar town revealed that characteristics like occupation, religion and ethnicity did not show a significant association with preference of pill, injectables and Norplant (22).

The result of our study showed that education has a positive influence in family planning information which was $65 \%$ among illiterates versus $90.9 \%$ among those educated up to primary, and those of secondary and tertiary share $100 \%$. Those women who had at least primary education had good propensity to modern family planning methods than illiterates. It seems that once a woman enters the school system, her attitude towards family planning changes. Additionally, greater awareness on alternative life styles could be achieved through education (23). Also a cross sectional survey conducted to assess women's role in family planning in Jimma town showed that the more educated the women is the more likely she is to have the final say on family planning (13).

The source of information for the majority 145(70.4\%) were from the health institution, followed by mass media and friend accounts for $50(24.3 \%)$ and 44(21.4\%) respectively. This result is somewhat higher when compared with cross sectional study conducted in Bahir Dar and per-urban area revealed which showed that frequently mentioned sources of information were mass media (69.8\%) followed health institution and friends which were $66 \%$ and $14.4 \%$ respectively(22)

In our study 59\% of respondents had used some modern Family Planning methods and $46.2 \%$ of the respondents were using FP methods at the time of the study, ever use of FP among urban dwellers $73 \%$ and $45.7 \%$ among rural dwellers; this result showed similar cross sectional study conducted in Bahir Dar town and per urban 
to assess knowledge attitude and practice of Family Planning which was $19.5 \%$ Vs $14.5 \%$ (factors..). Among ever users $13.8 \%$ switched to other method while $21.6 \%$ stopped to use and $65.5 \%$ continued to use this result showed the comparable with across sectional study on quality of Family Planning services and perception of clients in Sodo town, Southern Ethiopia, which showed that about $75 \%$ of women had used Family Planning method in the past, of which ever used, $41.6 \%$ stopped to use, $8.4 \%$ switched to other methods and $49.6 \%$ continued use of the method at time of survey (13)

Our result showed that socio demographic variables like educational status has positive effect on practice of family planning methods which were $46 \%$ among illiterate Vs $52.9 \%$ among those attained at least primary school. Similarly a survey conducted in Jimma town showed the fact that the more educated the women is the more likely she is to have the final say on family planning (13).

Our study showed that In our study $173(78.3 \%)$ respondents had six and less family members $48(21.7 \%)$ respondents had more than six family members. This result is similar with survey of Guttmacher institute of Ethiopia which showed that the average family size is slowly declining from 6.4 children per woman in 1990, to 5.9 in 2000 , to 5.4 in 2005.

Our result showed that the mean age at marriage in generally was found to be 18 years and for that of urban dwellers was 21 years while for rural was 17 years. But across sectional study conducted in Hawassa town, SNNPR to assess magnitude and determinants of un met need for family planning showed that the mean age at marriage in urban and rural were found to be 18 and 16 years respectively(16).

This study conducted among child bearing age of women of Wonago health center, Gedeo zone, Southern, Ethiopia to assess knowledge attitude and practice towards modern family planning methods is concluded as:

- Socio demographic factors like residence and educational status are associated with information about family planning and ever practice of it.

- Majority of the respondents knew at least one type of modern family planning methods.

- The major source of information on family planning is from health institutions.

- Educational status at primary school and above is positively associated with information on family planning and ever use of it.

- Most of the respondents were found to have favorable attitude towards modern family planning methods.

- More than half of the respondents had ever practiced some modern FP methods.

- The mean age at marriage of the urban dwellers is higher than that of the rural.

- Inject able FP method was the most commonly preferred method followed by or plant.

\section{Acknowledgment}

I would like to express my gratitude to all data collectors for their unreserved help and facilitation.

My heartfelt thanks also goes to Rift valley University for giving me this chance to do this research work. Finally, I am greatly indebted to my wife Netsanet Desalegne she gave me courage deserves my utmost gratitude for helping me to do this Research work.

\section{References}

1. Hofmann. AM. Grey danns DE. Adolescent Medicine second edition 1989: 1-355 (1-20)

2. WHO, Adolescent friendly health services 2003:3

3. Gebeyehu Mekonen and Mekdes Alemu, Adolescent sexuality and contraceptives in Addis Ababa, Nazreth and Hawassa 1995 (unpublished).

4. Konjit kifelew. Some socio demographic determinants and consequences of female adolesecents sexual behavior in Addis Ababa. Msc thesis in Demography, AAU:1998

5. Firehiwot Birhane .Health problems and services preferences of school adolescents of Addis Ababa With emphasis on Reproductive Health.MPH thesis, AAU, 2000.

6. Ethiopia Demographic and Health survey, central statistical Agency, Addis Ababa Ethiopia, 2011

7. Int Nurs Rev. 2006.53 (4) 269-276

8. Studies in Family planning Vol 5- No 11 (Nov 1974), PP344-348

9. Hawassa university department of Obstetrics and Gynecology, lecture Note for health science students, $1^{\text {st }}$ edition, 2006.

10. Lily P.Kiki, Marjorie Brahanss singer, the introduction of community based family planning service in Rural Mali. The katibou Family health project, working paper No2, 1993.

11. Lily.P.kiki, Estate E.Quain and susans Richiedic, Reaching urban women with family planning services in Ahmadabad, India, Working paper No3,1993.

12. Uffo Bonga,Wogasso ,Formative Evaluation of Quality of family planning services and perception of clients the case of soddo Town, Wolayta Zone, SNNPRS,2005.

13. Birhan Research and development consultancy, KAP in Family planning in Amhara, Oromiya, SNNPR and 
Tigray regions of Ethiopia, 2004.

14. Hanna Yilma. Modern contraceptive prevalence and KAP study among women of reproductive age group (15-49) in Bahir Dar town and pre-urban areas, Northern western Ethiopia, 2002.

15. Rokia Aidahis Aberra, the role of emergency contraception on women's reproductive health: Awareness Demand and Accessibility issues in Addis Ababa, 2007.

16. Sahile site, Assessment of the Magnitude and determination of unmet need for FP among currently married women in urban and per urban community in Hawassa, Southern Ethiopia, 2003.

17. Alemu kebede, Assessment of Quality of care in Family planning services with particular Reference to East Gojjam Administrative zone, Amhara National Regional state, 2007.

18. Atsede Desta, Emergency contraceptives: KAP among Bahir Dar University female students, north western Ethiopia, 2007.

19. Misrak Mohammed, Woman's Role in family planning decision -making the case of Jimma town, 2007.

20. WWW. Owen org./blog/ 3706, Sept 23, 2010.

21. Yigzaw kebede, emergency contraceptives knowledge and practice of Gonder University students, North west in Ethiopia, 2006

22. Hanna Yilma, Modern contraceptive preference and KAP study among women of Reproductive age group (15-49) in Bahir Dar town and pre-urban area, North West Ethiopia, 2002.

23. Tsigereda Gadisa, Barriers to Use of Contraceptives Among Adolescents in the City of Addis Ababa, MPH Thesis, April 2004 